\title{
Ceramic Cr:ZnS Laser Mode-Locked by Graphene
}

\author{
Nikolai Tolstik $^{1 *}$, Evgeni Sorokin ${ }^{2}$, and Irina T. Sorokina ${ }^{1}$. \\ Department of Physics, Norwegian University of Science and Technology, N-7491 Trondheim, Norway, E-mail: nikolai.tolstik@ntnu.no \\ Institut für Photonik, TU Wien, Gusshausstrasse 27/387, A-1040 Vienna, Austria.
}

\begin{abstract}
We report a high-power graphene mode-locked ceramic Cr:ZnS-laser, producing $3.9 \mathrm{~nJ}$, $140 \mathrm{fs}$ pulses with $45 \mathrm{~nm}$ spectral bandwidth at $270 \mathrm{MHz}$ repetition rate, at output power for the first time exceeding $1 \mathrm{~W}$ level.

(C2014 Optical Society of America OCIS codes: 140.4050 Modelocked Lasers; 140.3070 Infrared and farinfrared lasers, 140.3580 Lasers, solid-state
\end{abstract}

\section{Introduction}

Femtosecond Cr:ZnS lasers made a great progress in the last few years. The realization of different mode-locking mechanisms, such as SESAM [1,2], Kerr-lens [3,4], and, recently, graphene [5,6] and carbon nanotubes [7] resulted in a wide range of the implementations and output parameters, reaching $44 \mathrm{fs}$ pulse duration [5] and $1 \mathrm{~W}$ average output power [6]. These remarkable results have been mostly achieved using the monocrystalline Cr:ZnS, which is not easy to produce. At the same time the more technological polycrystalline Cr:ZnS was reported to show a very good performance in the continuous-wave regime [8]. In this paper we report the first realization of a graphenemodelocked femtosecond $\mathrm{Cr}: \mathrm{ZnS}$ ceramic laser with excellent characteristics, made possible by the simultaneous implementation of the purposely designed chirped- and graphene mirrors.

\section{Experimental setup}

The experimental setup is shown in Fig. 1. The pump beam of the Er-fiber laser at $1.61 \mu \mathrm{m}$ was focused by the 40$\mathrm{mm}$ AR-coated lens to a $60 \mathrm{x} 130-\mu \mathrm{m}$ spot on the crystal. The X-folded near-symmetrical astigmatically compensated four-mirror cavity had a round-trip length of about $1.1 \mathrm{~m}$. Active element (2-mm-thick diffusion-doped Cr:ZnS ceramics) was positioned at Brewster angle between the concave mirrors having $50 \mathrm{~mm}$ ROCs. The crystal absorbed about $85 \%$ of the pump emission. Graphene saturable absorber was deposited on the surface of the flat high-reflector mirror thus forming graphene-based saturable absorber mirror. The cavity mode was additionally focused to graphene saturable absorber by $\mathrm{ROC}=100 \mathrm{~mm}$ concave chirped mirror. No active cooling was necessary at this power level. We used an output coupler with $18 \%$ transmittance. The compensation of the group-delay dispersion (GDD) was achieved by the single chirped mirror (CM) in a double-bounce configuration.

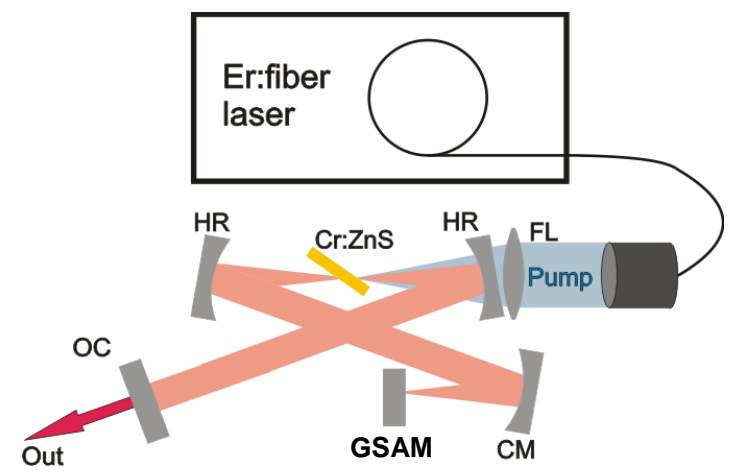

Figure 1. Experimental setup of the graphene-mode-locked ceramic Cr:ZnS laser. FL-focusing lens, HR - high reflector mirror, $\mathrm{CM}$ - chirped mirror, GSAM - graphene-based saturable absorber mirror, OC - output coupler.

\section{Results and discussion}

The laser was self-starting and was operated in the anomalous dispersion regime (also called "negative dispersion regime" or "soliton regime") with dispersion around $-500 \mathrm{fs}^{2}$ per cavity roundtrip. The laser produced up to $1.05 \mathrm{~W}$ of average output power on the cavity fundamental frequency of $270 \mathrm{MHz}$, providing the maximum pulse energy of 
$3.9 \mathrm{~nJ}$. The pulse energy was limited by the pump power available, but according to our previous results with singlecrystalline Cr:ZnS [3], we are quite close to the pulse breakup limit due to third-order optical nonlinearity of the active crystal. The pulse duration shortened with the increase of the pump power, reaching the shortest duration of $140 \mathrm{fs}$ at the maximal output power. The laser spectrum centered at $2.35 \mu \mathrm{m}$ had a bandwidth of $45 \mathrm{~nm}$ FWHM. The pulses were close to the transform-limited with time-bandwidth product of 0.336 . The dispersion curve, laser spectrum and autocorrelation function are plotted in Fig. 2.
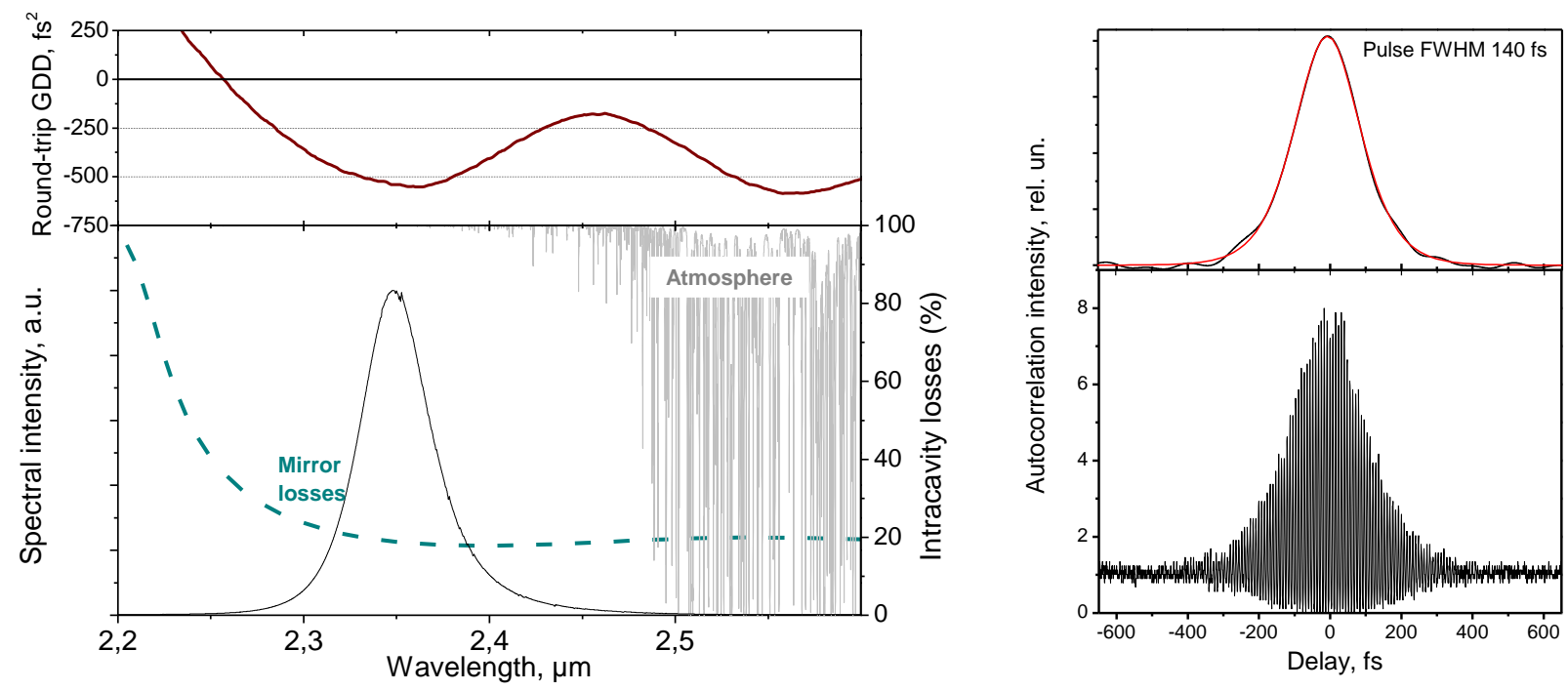

Fig. 2. The intracavity dispersion and laser spectrum of the graphene-mode-locked ceramic Cr:ZnS laser, as well as OC losses and atmosphere absorption (left); interferometric autocorrelation trace and FT-filtered intensity autocorrelation trace with sech ${ }^{2}$ fitting (right).

We have reported the KLM Watt-level femtosecond Cr:ZnS laser recently [4]. This configuration of the laser has a number of advantages. The implementation of the ceramic active element allows to eliminate crystal growth difficulties of monocrystalline Cr:ZnS. Mode-locking by graphene-based saturable absorber mirror results in a reliable self-starting and simplified cavity adjustments. Shortening the cavity and increasing the pump absorption efficiency allow reaching high output power in a reliable single-pulse regime with a controllable pulse repetition rate. We have not observed any damage of either active element or passive shutter during the experiments. Further shortening the pulse duration requires optimization of the mirror designs for fine-tuning the intracavity GDD.

To conclude, we have demonstrated for the first time ceramic Cr:ZnS laser mope-locked by graphene-based saturable absorber mirror. The laser provided average output power over $1 \mathrm{~W}$ with pulse duration of $140 \mathrm{fs}$ and repetition frequency of $270 \mathrm{MHz}$. The laser spectrum reached $45 \mathrm{~nm}$ bandwidth and was centered at $2.35 \mu \mathrm{m}$. We have demonstrated a simple and feasible approach for reliable femtosecond laser in the mid-IR, which should be interesting for a number of applications.

This work is supported by the NFR project FRITEK/191614, NANO 2021 project N219686, FWF project P24916N27, and the FP7 project GRAPHENICS.

\section{References}

1. E. Sorokin, N. Tolstik, I. T. Sorokina, "Femtosecond operation and self-doubling of Cr:ZnS laser," in Nonlinear Optics 2011, (Optical Society of America, 2011), paper NThC1.

2. E. Sorokin, N. Tolstik, K. Schaffers, I. T. Sorokina, "Femtosecond SESAM-modelocked Cr:ZnS laser," Opt. Express, 20, 28947 (2012).

3. N. Tolstik, E. Sorokin, and I. T. Sorokina, "Kerr-lens mode-locked Cr:ZnS laser," Opt. Lett. 38, 299 (2013).

4. N. Tolstik, I. T. Sorokina, and E. Sorokin, "Watt-level Kerr-Lens Mode-Locked Cr:ZnS Laser at $2.4 \mu \mathrm{m}$," in CLEO: 2013, OSA Technical Digest (online) (Optical Society of America, 2013), paper CTh1H.2

5. N. Tolstik, A. Pospischil, E. Sorokin, and I. T. Sorokina, "Graphene mode-locked Cr:ZnS laser with 44 fs pulse duration" in Mid-Infrared Coherent Sources, OSA Technical Digest (CD) (Optical Society of America, 2013), paper MW1C.1

6. N. Tolstik, E. Sorokin, and I. T. Sorokina, "Graphene mode-locked Cr:ZnS Chirped-Pulse Oscillator" in Mid-Infrared Coherent Sources, OSA Technical Digest (CD) (Optical Society of America, 2013), paper MW1C.2

7. N. Tolstik, O. Okhotnikov, E. Sorokin, I. Sorokina, "Femtosecond Cr:ZnS laser at $2.35 \mu \mathrm{m}$ mode-locked by carbon nanotubes", SPIE proc. To be published (2014).

8. I.S. Moskalev, V.V. Fedorov, and S.B. Mirov, “10-Watt, pure continuous-wave, polycrystalline Cr:ZnS laser”, Opt. Express 17, 2048 (2009). 